\title{
Einfach komplex? \\ Die Übersetzung politikwissenschaftlicher Komplexität in die Gesellschaft
}

\author{
Janne Mende $(\mathbb{1}) \cdot$ Stefan Müller
}

Online publiziert: 4. August 2020

(C) Der/die Autor(en) 2020

Zusammenfassung Der Artikel diskutiert die Herausforderungen des Transfers politikwissenschaftlicher Komplexität in die Gesellschaft. Wir unterscheiden dafür zwischen den drei idealtypischen Modellen a) einer Vereinfachung von Komplexität, b) der Abbildung von Komplexität sowie c) der Übersetzung von Komplexität. Die Anforderungen an das dritte Modell einer Übersetzung politikwissenschaftlicher Komplexität entwickeln wir anhand fachdidaktischer Diskussionen der Politischen Bildung, die wir Subdisziplinen-übergreifend mit dem Beispiel unternehmerischer Menschenrechtsverantwortung aus der Global-Governance-Forschung verbinden. Der reflexiven Politischen Bildung entnehmen wir Multiperspektivität, die Offenlegung und Begründung normativer Maßstäbe, eine subjektorientierte Mündigkeit der Adressat/-innen und das Aushalten von Spannungsfeldern, um Bildungsprozesse zu organisieren. Die Möglichkeiten und Grenzen der Übersetzung von Komplexität zeigen wir anhand der Weiterentwicklung einer menschenrechtlichen Unternehmensverantwortung auf globaler Ebene auf. Dieses Feld ist von Spannungen zwischen einem hohen Grad an (rechtlicher, wirtschaftlicher und politischer) Komplexität und einem Bedürfnis nach einfachen Antworten gekennzeichnet. Eine zentrale Stärke der Übersetzung von Komplexität skizzieren wir darin, dass sie einen Startpunkt für gesellschaftliches Denken und Handeln anbietet, statt dieses durch Dichotomien, Vorenthalten von Wissen, Überforderung oder vorgegebene Antworten abzuschneiden. So kann die Übersetzung von politikwissenschaftlicher Komplexität gesellschaftliche Denk- und Handlungsmöglichkeiten ausbauen.

PD Dr. J. Mende ( $\varangle) \cdot$ PD Dr. S. Müller

Institut für Politikwissenschaft, Justus-Liebig-Universität Gießen, Gießen, Deutschland

E-Mail: janne.mende@sowi.uni-giessen.de

PD Dr. S. Müller

E-Mail: stefan.mueller@sowi.uni-giessen.de 


\section{Simply complex? The translation of complexity from political science into society}

Abstract The paper discusses the challenges of transferring complexity from Political Science into society. We suggest three ideal-typical models of a) the reduction of complexity, b) the reproduction of complexity, and c) the translation of complexity. In order to develop our model of a translation of complexity, we connect insights from Civic Education with a case study from Global Governance research, thereby connecting the two sub-disciplines. The perspective of a reflexive Civic Education provides us with the elements of multiperspectivity, open normativity, autonomy, productive contradictions and the organization of self-thinking. The global governance field of business responsibility for human rights illuminates both the chances and the limits of transferring complexity into society, as this field is marked by a high degree of (political, economic and legal) complexity as well as by the need for straightforward answers. We suggest the model of translating complexity to bring forward complexity as a starting point for societal thinking and agency, rather than to limit the latter through dichotomies, reduction of knowledge, overload or prescriptions. Ultimately, the translation of complexity can strengthen knowledge, thinking and agency in society.

\section{Einleitung}

Politikwissenschaftliche Analyse und deren Transfer und Wirkung in die Gesellschaft hinein steht vor Herausforderungen. Eine der zentralen Herausforderungen scheint in der Lücke zwischen komplexen Wissens- und Forschungszusammenhängen einerseits und einfachen Darstellungen in deren Transfer in die Gesellschaft andererseits zu bestehen. Unserer Frage nach diesem Transfer liegt ein breites Verständnis von Gesellschaft zugrunde, das sowohl öffentliche als auch politische Debatten umfasst. Diesen (ihrerseits heterogenen) Feldern ist die Annahme gemeinsam, dass Komplexität vereinfacht werden müsse, um gehört, verstanden bzw. angewendet zu werden. Mit dieser Annahme gehen allerdings auch problematische Effekte einher. Zum einen kann Vereinfachung gegenstandsunangemessen sein, weil Wissenschaft und Gesellschaft trotz ihrer gegenseitigen Verwiesenheit aufeinander unterschiedliche Logiken und Wissensformen aufweisen. ${ }^{1}$ Zum anderen suggeriert Vereinfachung vermeintlich einfache Lösungen für komplexe Probleme. Spätestens dann, wenn die Vereinfachung nicht mehr eingehalten werden kann, weil widersprüchliche Annahmen auftreten und beide begründbar sind, droht den politikwissenschaftlichen Wissensformen auch ein Legitimitätsverlust in der Gesellschaft, ganz zu schweigen von den politischen und gesellschaftlichen Effekten wie der Stärkung populistischer Annahmen, die sich aus dem Angebot vereinfachender Lösungen speisen.

\footnotetext{
1 Darauf gründet auch das Feld der Wissenschaftskommunikation. Es verdeutlicht, dass die Vermittlung wissenschaftlicher Forschung anderer Formen, Sprache und Aufbereitung bedarf als der wissenschaftsinternen. Bspw. Dernbach et al. (2013); Bonfadelli et al. (2017).
} 
Ein mögliches Gegenmodell besteht darin, auf eine unveränderte Abbildung von Komplexität abzuzielen. Auch dieses Modell weist allerdings Grenzen auf, weil es die Eigenlogiken von Politikwissenschaft und Gesellschaft übergeht und auf die Seite der Wissenschaft hin auflöst. Damit können die Problematiken verbunden sein, in Unübersichtlichkeit oder Relativismus überzugehen, in Detailfragen verhaftet zu bleiben und den Anschluss, die Relevanz und Wirkmächtigkeit von Politikwissenschaft in der Gesellschaft einzuschränken.

Vor dem Hintergrund dieser Vereinseitigungen diskutieren wir im Folgenden Möglichkeiten, Komplexität in die Gesellschaft zu vermitteln, ohne sie zu reduzieren. Wir unterscheiden mithin zwischen den drei idealtypischen Modellen a) der Vereinfachung von Komplexität, b) der Abbildung von Komplexität sowie c) der Übersetzung von Komplexität.

Für unsere Diskussion verbinden wir Subdisziplinen-übergreifend Perspektiven aus der Global-Governance-Forschung mit der Fachdidaktik der Politischen Bildung. Am Beispiel der Unternehmensverantwortung für Menschenrechte (Kap. 2) illustrieren wir den gesellschaftlichen und politischen Wunsch nach einfachen Lösungen, der sich an dichotomen Zuordnungen orientiert, und das Gegenmodell einer Abbildung von Komplexität. Anhand der Grenzen, aber auch der Möglichkeiten beider Modelle stellen wir die Anforderungen an eine dritte Umgangsmöglichkeit heraus, die die Komplexität politikwissenschaftlicher Analyse nicht verkürzt, sondern beibehält - aber so übersetzt, dass die Komplexität für alle Beteiligten nachvollzogen werden kann.

Diese Anforderungen entwickeln wir anhand fachdidaktischer Diskussionen in der Politischen Bildung (Kap. 3). Der reflexiven Politischen Bildung entnehmen wir die Ansprüche der Multiperspektivität, die Offenlegung und Begründung normativer Maßstäbe und eine subjektorientierte Mündigkeit der Adressat/-innen für die Organisation von politischen Bildungserfahrungen. Die solchermaßen gekennzeichnete reflexive Politische Bildung zielt darauf ab, Spannungsfelder in ihren Widersprüchlichkeiten anzuerkennen und für Bildungserfahrungen auszugestalten.

Diese Ansprüche bilden die Grundlage für unser Modell der Übersetzung von politikwissenschaftlicher Komplexität in die Gesellschaft (Kap. 4). Dieses Modell kann Widersprüchlichkeiten (genauer: innere Vermittlungsverhältnisse) ebenso benennen und aushalten wie die unterschiedlichen Logiken der getrennten und doch verbundenen Felder. Die mögliche Ausgestaltung der Übersetzung von Komplexität zeigen wir anhand des Beispiels der Unternehmensverantwortung für Menschenrechte auf.

Abschließend (Kap. 5) verdeutlichen wir die Stärken und die Problematiken, die alle drei Modelle aufweisen. Eine zentrale Stärke der Übersetzung von Komplexität besteht darin, dass sie einen Startpunkt für gesellschaftliches Denken und Handeln anbietet, statt dieses durch Dichotomien, Vorenthalten von Wissen oder vorgegebene Antworten abzuschneiden. Insgesamt geht es darum, dass die Übersetzung von Komplexität sowohl gesellschaftliches Wissen als auch gesellschaftliche Denk- und Handlungsmöglichkeiten ausbauen und vertiefen kann. 


\section{Der Transfer von Komplexität am Beispiel der Unternehmensverantwortung für Menschenrechte}

Die Herausforderungen, mit denen der Transfer politikwissenschaftlicher Komplexität in die Gesellschaft konfrontiert sein kann, zeigen wir exemplarisch am Feld der Unternehmensverantwortung für Menschenrechte aus der Global-Governance-Forschung auf. Dieses Feld ist von einer ausgeprägten Verflechtung gesellschaftlicher, politischer und akademischer Debatten sowie vom Wunsch nach einfachen, im Sinne von umsetzbaren, handhabbaren und praktikablen Lösungen bestimmt. Nach einer kurzen Einführung in das Feld diskutieren wir im Folgenden die Anschlussmöglichkeiten und die Grenzen der Reduktion sowie der Abbildung von Komplexität.

Das Menschenrechtsregime bildet einen völkerrechtlichen, gesellschaftlichen und politischen Referenzrahmen, das normativ nahezu universell geteilt wird (Mende 2019), wenngleich es durch deutliche Lücken geprägt ist. Diese Lücken gehen auch auf Entwicklungen der Globalisierung und der Global Governance zurück. Die hier entstehenden Handlungsmöglichkeiten und Machtformen von nichtstaatlichen Akteuren stehen dem klassisch auf Staaten als Verantwortungsakteure ausgerichteten Menschenrechtsregime gegenüber (Mende 2020b). Hier zeichnet sich ein fundamentaler struktureller Widerspruch ab. Vor diesem Hintergrund hat sich eine globale Debatte entwickelt, die darauf abzielt, auch Unternehmen als nichtstaatliche Akteure in die menschenrechtliche Verantwortung zu nehmen (Noortmann et al. 2015; Clapham 2006; Deva und Bilchitz 2013). Diese Debatte hat seit den 1990er Jahren international an Resonanz gewonnen und konnte bedeutende Teilerfolge erringen - etwa mit der UN-Plattform des Global Compact, den UN-Leitprinzipien für Wirtschaft und Menschenrechte und der Bedeutung von Menschenrechtsverantwortung für Unternehmens-Governance und Corporate Social Responsibility (Scherer und Palazzo 2011; Ramasastry 2015). Gleichzeitig ist das Feld von grundlegenden Kontroversen um die Ausgestaltung der Regulierungsinstrumente geprägt (von Bernstorff 2011; Addo 2014; Mende 2017; Černič und Carrillo Santarelli 2018). Diese Kontroversen sind gekennzeichnet von einem Spannungsfeld zwischen einem hohen Grad an (rechtlicher, wirtschaftlicher und politischer) Komplexität einerseits und der Notwendigkeit, diese Komplexität politisch bearbeitbar zu halten, praktisch $\mathrm{zu}$ operationalisieren und gesellschaftlich zu vermitteln andererseits. Die folgende Diskussion des Transfers dieser Komplexität beruht auf einem Forschungsprojekt, das die Entwicklung unternehmerischer Menschenrechtsverantwortung im Rahmen der Vereinten Nationen untersucht. ${ }^{2}$

\subsection{Vereinfachung von Komplexität}

Das Versprechen einer Vereinfachung von Komplexität wird vor allem mit einer breiten gesellschaftlichen Aufmerksamkeit verbunden, die zur Durchsetzung politischer und rechtlicher Antworten führen könne und die Anwendung handhabbarer

\footnotetext{
2 Das Forschungsprojekt „Business Actors beyond Public and Private: Authority, Legitimacy and Responsibility in the United Nations Human Rights Regime“ (BAPP) wird gefördert durch die Deutsche Forschungsgemeinschaft (DFG), Projektnummer 398306144.
} 
Lösungen erlaube. Diese Perspektive zeigt sich insbesondere in drei dichotomen Anschlussmöglichkeiten, die jeweils einen gegen den anderen Standpunkt ausspielen: a) öffentliche oder private Menschenrechtsverantwortung, b) für oder gegen einen neuen völkerrechtlichen Vertrag, c) gute oder böse Unternehmen.

a) Die Dichotomie öffentliche oder private Menschenrechtsverantwortung ergibt sich aus der völkerrechtlichen Konstellation, die zwischen staatlichen (im Sinne des Völkerrechts öffentlichen) und nichtstaatlichen (privaten) Akteuren unterscheidet. In dieser Dichotomie haben Staaten die Pflicht, Menschenrechte zu achten, zu schützen und zu gewährleisten. Das schließt aktive und positive Pflichten ein. Private Akteure haben demgegenüber die negative Verantwortung, Menschenrechte zu achten, indem sie Menschenrechte nicht verletzen. Die Gewährleistung dafür übernimmt wiederum der Staat, der auch für das Handeln privater Dritter in seiner Jurisdiktion oder seinem Territorium verantwortlich ist.

Die Entwicklung der unternehmerischen Menschenrechtsverantwortung stellt diese dichotome Trennung zunächst einmal in Frage. Gleichzeitig bewegt sie sich jedoch innerhalb dieses Rahmens und wird mit dessen Maßstäben gemessen. Paradigmatisch dafür stehen die sogenannten Draft Norms, ${ }^{3}$ mit deren Entwurf ein UN-Gremium versucht hat, Unternehmensverantwortung für Menschenrechte auf völkerrechtlicher Ebene zu etablieren. Die Draft Norms scheiterten 2003 insbesondere an der Kritik, dass sie die Pflichten von Unternehmen und Staaten nahezu gleichsetzen würden, indem sie Unternehmen direkte und positive Pflichten auferlegen wollten (Fitzgerald 2019; Miretski und Bachmann 2014) - mit anderen Worten: dass sie eine öffentliche statt einer privaten unternehmerischen Menschenrechtsverantwortung anstrebten. Die daraufhin entwickelten UN-Leitprinzipien für Wirtschaft und Menschenrechte (2011) werden als Meilenstein für die Entwicklung unternehmerischer Menschenrechtsverantwortung behandelt. Mit dem Modell der Komplementarität zwischen staatlicher und unternehmerischer Verantwortung bieten sie durchaus Möglichkeiten, eine Dichotomie von „entweder-oder“ zu überschreiten (Mende 2017). Jedoch verbleiben sie auch innerhalb des dichotomen Rahmens von öffentlicher oder privater Verantwortung, denn sie sind ein normativer, aber rechtlich nicht bindender Appell an Unternehmen, im Rahmen einer negativen Verantwortung Menschenrechtsverletzungen vorzubeugen und zu adressieren (Ruggie und Sherman 2017). Jenseits der Diskussion, ob die Leitprinzipien ihre Ansprüche erfüllen können (Ruggie 2018) oder ob sie zu lückenhaft bleiben (Muchlinski 2012; Deva und Bilchitz 2013), geht es uns an dieser Stelle darum, auf die Einschränkungen zu verweisen, die mit einer dichotomen Ordnung in der Vereinfachung von Komplexität verbunden sind: Es bleibt nur die Möglichkeit, entweder Unternehmenspflichten mit Staatenpflichten gleichzusetzen, was zu gravierenden demokratietheoretischen und völkerrechtlichen Problematiken führt, oder die unternehmerische Menschenrechtsverantwortung bleibt privat und somit lückenhaft, mit erheblichen Konsequenzen für die Betroffenen von Menschenrechtsverletzungen.

\footnotetext{
3 Draft Norms on the Responsibilities of Transnational Corporations and Other Business Enterprises with regard to Human Rights: Draft Norms/submitted by the Working Group on the Working Methods and Activities of Transnational Corporations pursuant to resolution 2002/8, U.N. Doc. E_CN.4_Sub.2_2003_12EN (2003). https://digitallibrary.un.org/record/498842.
} 
b) Die Unzufriedenheit mit solchen dichotomen Standpunkten führte $2014 \mathrm{zu}$ einem erneuten Versuch, ein völkerrechtlich bindendes Instrument in Bezug auf Unternehmen und Menschenrechte zu entwickeln: zum sogenannten UN-Vertragsprozess. ${ }^{4}$ Insbesondere während seiner Gründungsphase sowie in seinen Anfangsjahren war die Diskussion des UN-Vertragsprozesses stark von einer dichotomen Positionierung entweder für oder gegen den Vertrag geprägt. Der UN-Vertragsprozess galt als Gegenmodell zu den bis dahin etablierten UN-Leitprinzipien, die auf einem breiten Konsens beruhen, und somit auch als Angriff auf diesen Konsens (Tuttle 2015). Entsprechend aufgeladen und politisiert war in dessen Anfangszeit die Haltung gegenüber dem UN-Vertragsprozess, die zu einem dichotomen Freund-Feind-Denken zugespitzt wurde: Wer für den Erhalt des existierenden Konsensus war, musste gegen den UN-Vertragsprozess sein - und umgekehrt, so die dichotome Logik. Diese Ausgangsannahmen unterminieren allerdings nicht nur politikwissenschaftliche Komplexität, sondern auch die gesellschaftlichen und politischen Voraussetzungen für internationale Kooperation im Umgang mit widersprüchlichen Perspektiven. Dafür sind Deliberation, Argumentation, Abwägen und differenzierte, auch ergebnisoffene Auseinandersetzung mit dem Gegenstand notwendig.

c) Der Wunsch, sich dem Feld der Unternehmensverantwortung für Menschenrechte durch eindeutige Positionen zu nähern, äußert sich auch in gesinnungsethischen, moralischen bzw. politischen Klassifizierungen von Unternehmen als ,gut“ oder als ,schlecht“ (bzw. in der jeweils präferierten Terminologie). Wird der Charakter von Unternehmen als ,gut“ vorausgesetzt - beispielsweise, weil ihre Investitionen gesellschaftliche Entwicklung ermöglichen und damit (scheinbar) allen zugutekommen würden -, dann müssen sie als Partner der Regulierung und der Global Governance behandelt werden. Wenn Unternehmen als ,schlecht“ proklamiert werden - etwa, weil sie strukturell auf Profitmaximierung abzielen würden -, dann liegen Forderungen nach einer weitestmöglichen Regulierung und Sanktionierung (oder gar Abschaffung) nahe. In der Praxis kommt solchen Forderungen insbesondere in den Fällen Bedeutung zu, in denen ein Unternehmen direkt und unmittelbar Menschenrechte verletzt. Allerdings bilden diese Fälle nur einen Teilbereich der Unternehmensverantwortung für Menschenrechte ab. Herausfordernder ist bereits die in den UN-Leitprinzipien adressierte Komplizenschaft oder die Zusammenarbeit mit anderen Akteuren, die Menschenrechte verletzen (UN 2011: §13, 17). Noch komplexer ist die Frage nach unternehmerischen Einflussmöglichkeiten auf andere menschenrechtsverletzende Akteure - wenn Unternehmen Menschenrechte nicht selbst verletzen, aber etwas dagegen tun könnten (Wood 2012). Mit der Frage, inwiefern Unternehmen auch für andere - gar staatliche - Akteure verantwortlich sein können oder sollen, gerät eine dichotome Unterteilung zwischen ,guten“ oder ,schlechten“ Akteuren an ihre Grenzen. Abhilfe- und Verantwortungsmodelle kommen hier mit einer ,entweder/oder"-Sortierung nicht weiter.

\footnotetext{
42014 gründete der UN-Menschenrechtsrat auf Initiative von Ecuador und Südafrika mit Unterstützung weiterer Staaten vorranging des globalen Südens sowie mit starker Befürwortung aus der globalen Zivilgesellschaft eine Arbeitsgruppe mit dem Mandat, einen entsprechenden Vertrag zu entwerfen. Die seitdem laufenden Diskussionen und die daraus resultierenden Entwürfe bilden den UN-Vertragsprozess.
} 
Insgesamt verweisen alle drei Fälle auf Grenzen. Eine zu Dichotomien vereinfachte Perspektive wird dem komplexen In-, Mit- und Gegeneinander von theoretischen Beschreibungen und empirischen Analysen kaum gerecht. Komplexität tritt hier als zweifache Herausforderung auf: sowohl auf der Ebene politikwissenschaftlicher Analyse als auch auf der Ebene gesellschaftlicher Umgangsmöglichkeiten.

Nun ist die hier skizzierte Suche nach einfachen Antworten jedoch nicht lediglich mit dem Verweis auf die Komplexität des Feldes vernachlässigbar oder gar ignorierbar. Vielmehr finden sich sogar gute Gründe für diese Suche. Sie spiegelt zuvörderst die völkerrechtliche Trennung zwischen privat und öffentlich wider, welche die Umgangsmöglichkeiten bereits dichotom strukturiert. Einen weiteren Grund bildet der handlungsstrategische und pragmatische Aspekt von Einfachheit in politischen Entscheidungsprozessen und in gesellschaftlichen Debatten. Politische Transformationen scheinen gerade dann gesellschaftlich breit thematisierbar und durchsetzbar zu sein, wenn sie einfach vermittelt werden können. Diese Gründe gilt es demnach in der Übersetzung politikwissenschaftlicher Komplexität zu berücksichtigen.

\subsection{Abbildung von Komplexität}

Vor dem Hintergrund der Verkürzungen einer Vereinfachung von Komplexität liegt eine zweite idealtypische Umgangsmöglichkeit darin vor, die politikwissenschaftliche Komplexität in ihrer Komplexität beizubehalten und sie in die Gesellschaft zu übertragen. Politikwissenschaftliche Komplexität wird dann für den gesellschaftlichen Transfer nahezu unverändert abgebildet. Der Anspruch an Komplexität führt dann allerdings geradewegs in die Verkomplizierung, die in Detailfragen ebenso wie in Abstraktionen erscheinen kann.

a) So kann die Dichotomie von öffentlicher oder privater Menschenrechtsverantwortung durch die Anreicherung von Komplexität rasch überschritten werden. Dann ergibt sich ein vielfältiges Tableau an Möglichkeiten: angefangen bei dem Hinweis, dass die Konzepte von privat und öffentlich nicht ausreichen, um aktuelle Entwicklungen zu erfassen, über Mischmodelle und Kombinationen beider Pole bis hin zur Forderung nach ihrer Auflösung und Ersetzung durch andere Bezugspunkte. Hinzu kommen Fragen nach der (völker-) rechtlichen Umsetzung: Welche Akteure können oder müssen welche Formen der Menschenrechtsverantwortung übernehmen? Wie können sie straf- oder zivilrechtlich verfolgt werden? Welche Jurisdiktionen und Instanzen sind unter welchen Umständen zuständig und können sie extraterritorial entscheiden? Wie können Entschädigungen und Wiedergutmachungen gegenüber den Betroffenen von Menschenrechtsverletzungen verwirklicht werden? All diese Fragen werden in der Politikwissenschaft (und in anderen Disziplinen) höchst unterschiedlich beantwortet. Das Problem ist Folgendes: Eine Auflösung von Dichotomien gewährleistet weder einen Transfer noch eine produktive Wirkung in die Gesellschaft hinein. Die Berufung auf Komplexität kann auch geradewegs in Unübersichtlichkeit münden.

b) In der Positionierung für oder gegen einen neuen völkerrechtlichen Vertrag besteht eine andere Form der Überschreitung von Dichotomien in den Hinweisen, die auf Einschränkungen, Verbesserungsvorschläge oder alternative Wege im UNVertragsprozess verweisen. Tatsächlich finden sich zahlreiche Stellungnahmen zum 
UN-Vertragsprozess, die statt eines Bekenntnisses für oder gegen den Vertrag zu anderen diskursiven Mitteln greifen. So rückt u. a. eine Auseinandersetzung mit einzelnen Punkten des Vertrags in den Mittelpunkt, aber auch eine rein deskriptive Bestandsaufnahme des Status Quo, die sich weiterer Positionierungen zum Vertrag entzieht. Die Anerkennung der Komplexität des Feldes spielt für diese argumentative Umstellung eine zentrale Rolle. Allerdings kann der Rekurs auf Komplexität auch instrumentell genutzt werden, wenn er nicht als Beitrag zur Deliberation über einen internationalen Vertrag eingesetzt wird, sondern als Strategie seiner Verhinderung. So führte die über Jahre hinweg uneindeutige Position der Europäischen Union im UN-Vertragsprozess dazu, dass zahlreiche Staaten der EU (einschließlich Deutschland) sich nicht positionierten, sondern die Haltung der EU abwarteten. Das bedeutete ein jahrelanges Hinauszögern der Entwicklung politischer und rechtlicher Umgangsmöglichkeiten mit unternehmerischer Menschenrechtsverantwortung. Während die EU ihre Kritik als Beitrag zur Deliberation verstanden wissen will, werfen Nichtregierungsorganisationen ihr eine bewusste Unterminierung des UNVertragsprozesses vor. Zudem bildet die Überkomplexität im Bereich von Lieferund Wertschöpfungsketten innerhalb der Debatten ein Argument, um gegen die weitere Regulierung von Unternehmensverantwortung zu argumentieren, da die Regulierung entweder zu lückenhaft verbleiben oder zu restriktiv ausfallen müsse. Mit solch einem hohen Grad an Komplexität, so das Argument in den Stellungnahmen insbesondere von Unternehmensverbänden, könne schlicht kein angemessener Umgang durch verbindliche Regeln gefunden werden.

c) Eine Überschreitung der dichotomen Unterteilung in gute oder schlechte Unternehmen ist mit mehreren Herausforderungen konfrontiert. Zum einen stellt sich das Problem der Verantwortungszuschreibung, die jenseits von Dichotomien sowohl kausal als auch nicht kausal, direkt oder indirekt, moralisch oder rechtlich, macht- oder ressourcenbasiert diskutiert werden kann (bspw. Wood 2012; Wettstein 2012; Hsieh 2015). Diese Modelle sind geprägt durch jeweils andere Antworten auf die zugrundeliegenden Fragen: Entsteht Unternehmensverantwortung erst dann, wenn Unternehmen direkt und kausal zu einer Menschenrechtsverletzung beitragen, oder auch aus ihren Fähigkeiten, Menschenrechte zu schützen? Was unterscheidet Unternehmen im letzteren Fall von öffentlichen, politischen und (im Idealfalle) demokratisch legitimierten Akteuren?

Zum anderen stellt sich die Frage nach der Bedeutung normativer Bezugnahmen. Das Modell der Abbildung von Komplexität strebt an, die Einfachheit der Dichotomie mitsamt ihrem vermeintlich einfachen normativen Maßstab zu überschreiten. Allerdings bildet im Feld der menschenrechtlichen Unternehmensverantwortung das Menschenrechtsregime durchaus einen etablierten und institutionalisierten Maßstab, der eine Differenz von „gut“ und ,schlecht“ markiert und die Maßstäbe für diese Unterscheidung offen legt. ${ }^{5}$ Selbst vor diesem Hintergrund jedoch kann unternehmerisches Handeln eine Gleichzeitigkeit von menschenrechtsstärkenden und menschenrechtsschwächenden Effekten aufweisen: etwa dann, wenn der Coca-Cola-Konzern für seinen Umgang mit gewerkschaftlichen Bewegungen oder für die gesundheitli-

\footnotetext{
5 Auch diese Komplexität könnte jedoch angereichert werden: Menschenrechte können einander widersprechen, bspw. im Bereich individueller versus kulturell-kollektiver Rechte (Mende 2015).
} 
chen Folgen seiner Produkte unter massiver Kritik steht und sich gleichzeitig umfassend an der Distribution von AIDS/HIV-Medikamenten in Tansania und anderen afrikanischen Ländern beteiligt, ${ }^{6}$ oder wenn in Räumen begrenzter Staatlichkeit die unternehmerische Bereitstellung von Infrastruktur, Bildung und Gesundheitsversorgung positive Effekte für Unternehmensmitarbeiter/-innen hat, aber zu einer eklatanten Verschlechterung der Lebensbedingungen der lokalen Bevölkerungsteile führt, die nicht für das Unternehmen arbeiten (Atal 2017). Wenn aber ,gut“ und „schlecht“ derart nah beieinander liegen, kann die Abbildung von Komplexität in einen Relativismus kippen, der keine normative Unterscheidungsmöglichkeit mehr bietet.

In allen drei diskutierten Beispielen werden Herausforderungen deutlich, die zu Problematiken führen können. Insgesamt kann die Abbildung von Komplexität eine Verkomplizierung und damit auch mangelnde gesellschaftliche und politische Anschlussmöglichkeiten nach sich ziehen. Ihre Stärke besteht allerdings darin, dass sie auch zu einer Multiperspektivität und zu einer Auflösung von Dichotomien führt. Statt einfacher Antworten in die eine oder die andere Richtung wird ein Antwortreigen aufgespannt, der eine Vielzahl an Möglichkeiten eröffnet. Damit geht einerseits die Gefahr einher, nicht nur dichotome Abgrenzungen aufzulösen, sondern auch darüberhinausgehende Unterscheidungsmerkmale preiszugeben. Andererseits findet sich hierin auch eine Stärke politikwissenschaftlicher Komplexität, die in die Übersetzung einbezogen werden kann.

\subsection{Zwischenergebnis: Das Ziel der Übersetzung von Komplexität}

Die empirische Diskussion der Modelle der Vereinfachung und der Abbildung von Komplexität verdeutlicht nicht nur Grenzen, sondern auch Lösungsmöglichkeiten: Das gesuchte Modell muss über dichotome Zuschreibungen hinausgehen und Multiperspektivität erlauben, ohne in Unübersichtlichkeit oder Relativismus überzugehen. Es muss eine normative Verortung erlauben, gleichzeitig muss es Offenheit für Deliberation und Argumentation ermöglichen. Komplexität soll nicht reduziert, sondern anerkannt werden - ohne dabei die Gründe für die Suche nach Vereinfachung außer Acht zu lassen.

Die Fachdidaktik der politischen Bildung diskutiert die Frage, wie fachwissenschaftliche Analysen so aufbereitet werden können, dass sie von Bildungsteilnehmer/-innen angemessen angeeignet und in ihrer Komplexität, ihrer Reichweite, aber auch im Blick auf ihre Größen und Grenzen eigenständig beurteilt werden können. Daher diskutieren wir im nächsten Schritt Möglichkeiten der Fachdidaktik, die wir für die Übersetzung politikwissenschaftlicher Komplexität heranziehen.

\footnotetext{
${ }^{6}$ https://www.theglobalfund.org/en/private-ngo-partners/delivery-innovation/coca-cola/. Letzter Zugriff: 14.07.2020.
} 


\section{Fachdidaktische Möglichkeiten der Übersetzung von Komplexität: Reflexive politische Bildung}

Die Politikdidaktik beschäftigt sich mit dem Transfer von komplexen fachwissenschaftlichen Analysen in der schulischen und außerschulischen Bildung. Im Anschluss an den Beutelsbacher Konsens stehen die Fragen nach einer angemessenen Übersetzung fachwissenschaftlicher Analyse in die didaktische Gestaltung von Lernumgebungen für Bildungsteilnehmer/-innen im Mittelpunkt. ${ }^{7}$ Allerdings bilden die institutionellen Bedingungen von Schulpflicht einen Rahmen, der für unsere Frage des Transfers politikwissenschaftlicher Komplexität in die Gesellschaft nicht unmittelbar übertragen werden kann. Im Folgenden werden vier ausgewählte Möglichkeiten der Politikdidaktik skizziert, um diese dann in einem weiteren Schritt in ein Modell der Übersetzung von Komplexität zu überführen, das jenseits der institutionellen Rahmenbedingungen der schulischen Politischen Bildung verortet ist.

Für die folgenden Überlegungen ist eine Voraussetzung grundlegend, an die die Übersetzung politikwissenschaftlicher Komplexität direkt anschließen kann: „Didaktik ist eben nicht ,Reduktion“, sondern ,Transformation " unterschiedlicher Wissensformen." (Behrmann et al. 2004, S. 347) Auf dieser Grundlage werden wir die Vereinfachung oder gar das Vorenthalten politikwissenschaftlicher Komplexität problematisieren und auf ein multiperspektivisches Aushalten unterschiedlicher Wissensformen abzielen.

\subsection{Das Überwältigungsverbot}

Für die schulische Politische Bildung bildet das Überwältigungsverbot im Anschluss an den Beutelsbacher Konsens eine gleichsam axiomatische Bezugnahme:

Es ist nicht erlaubt, den Schüler - mit welchen Mitteln auch immer - im Sinne erwünschter Meinungen zu überrumpeln und damit an der „Gewinnung eines selbständigen Urteils“ zu hindern. Hier genau verläuft nämlich die Grenze zwi-

\footnotetext{
7 Die heute unter dem Stichwort Beutelsbacher Konsens bekannten drei Grundsätze der Politischen Bildung gehen zurück auf ein Tagungsprotokoll von Hans-Georg Wehling, einem Mitarbeiter der Landeszentrale für Politische Bildung in Baden-Württemberg (Wehling 1977). Der Protokollant selbst wählte den Titel „Konsens à la Beutelsbach? Nachlese zu einem Expertengespräch“. Im Protokoll nehmen die heute als Beutelsbacher Konsens bekannten Grundsätze verhältnismäßig wenig Raum ein. Die Tagung war dominiert von grundsätzlichen Differenzen, die sich vor allem an parteipolitischen Auseinandersetzungen in der Gestaltung von Lehrplänen äußerten. Der Protokollant skizziert das folgendermaßen: „Ein Auftrag, einen Konsens - etwa in Form eines Lehrplanes - nun auch tatsächlich zu produzieren, war nicht gegeben. So kann es sich an dieser Stelle nur darum handeln, zu skizzieren, wo der Verfasser nach seinen zugegebenermaßen subjektiven - Eindrücken einen Konsens für möglich hält, einen Konsens zwischen so unterschiedlichen wissenschaftstheoretischen, politischen und auch didaktischen Positionen" (Wehling 1977, S. 179). Damit verschob Wehling in ebenso umsichtiger wie entscheidender Hinsicht das Feld der Auseinandersetzungen: Gemeinsamkeiten in den Unterschieden wurden gesucht, indem die Unterschiede in den parteipolitischen und wissenschaftstheoretischen Präferenzen beibehalten wurden. Auf der Ebene von Lehrplänen konnte sich damit den didaktischen Zugängen genähert werden, weil nun prominenter die unterschiedlichen Effekte von gesinnungsethischen, proklamatorischen und mündigkeitsorientierten Konzepten konturiert werden konnten - eine Differenz, die sich bis heute bewährt (Müller 2018).
} 
schen Politischer Bildung und Indoktrination. (Wehling 1977, S. 179, Hervor. im Orig.)

Das Überwältigungsverbot entstand in einer Zeit, in der gesellschaftspolitisch konträre Vorstellungen polarisiert aufeinanderprallten (Sander 2017, S. 61). Auch heute handelt es sich um grundsätzlich divergierende Vorstellungen des guten, des besseren gesellschaftlichen Zusammenlebens. Kompromissformeln gibt es an dieser Stelle nicht - und muss es auch nicht geben, denn Politik und Gesellschaft zeichnen sich gerade auch durch sich widersprechende Annahmen und Konzepte aus. „Konflikte mit politischem Gewicht sind in einer pluralistischen Gesellschaft notwendig und nicht vermeidbar. Gefährlich ist der nicht sichtbare, womöglich abgedrängte Konflikt." (Reinhardt 2018, S. 80).

Das Überwältigungsverbot bildet hier eine Lösungsfigur, die die Diskursivität und den Konflikt um politische Auseinandersetzungen nicht eindimensional zu Gunsten der einen oder der anderen Seite auflöst. Das mag zunächst unbequem sein, weil die eigenen Vorstellungen und Überzeugungen damit auch erheblich eingeschränkt und die eigenen guten Gründe relativiert werden. Aber aus dem Überwältigungsverbot lassen sich so weiterführende Überlegungen für die Übersetzung von Komplexität in die Gesellschaft gewinnen: Zum einen wird Politik als umkämpftes und umstrittenes Feld anerkannt und ausgehalten. Zum anderen wird die Eigenständigkeit von Bildungsteilnehmer/-innen genau darin gewahrt, dass die „Gewinnung eines selbständigen Urteils“ als normative Zielverstellung unterstützt und all die Formen von (manchmal gar nicht so) stillschweigender Indoktrination problematisiert werden.

Damit zeichnen sich zwei Merkmale für unser Modell der Übersetzung von Komplexität ab:

M1) Die Anerkennung und das Aushalten von Spannungsfeldern als Reaktion auf einen strukturell durch Konflikte geprägten Gegenstandsbereich - anstelle eines eindimensionalen Auflösens;

M2) die (normative) Orientierung an der Eigen- und Selbständigkeit der Bildungsteilnehmer/-innen - anstelle von (politischer) Indoktrination bzw. einer Haltung, die davon geprägt ist, für sie zu denken.

\subsection{Multiperspektivität}

Die zweite Lösungsfigur entnehmen wir dem politikdidaktischen Prinzip der Multiperspektivität, auch und gerade im Anschluss an den Beutelsbacher Konsens: „Was in Wissenschaft und Politik kontrovers ist, muss auch im Unterricht kontrovers erscheinen." (Wehling 1977, S. 179, Hervor. im Orig.) Dieser Anspruch geht mit erheblichen Herausforderungen einher: Welche Positionen sollen in einem multiperspektivischen Verfahren (nicht) berücksichtigt werden? Wenn nicht lediglich Positionierungen einbezogen werden sollen, denen die je eigene Perspektive (gerade noch so) zustimmen kann, besteht Multiperspektivität darin, entgegenstehende, divergierende und den eigenen Ansichten auch entschieden widersprechende Positionen einzubeziehen. 
Eine reflexive Politische Bildung geht dabei über Verfahren hinaus, in denen unterschiedliche politische Positionierungen schlicht additiv aneinandergereiht und nebeneinander gestellt werden. Zunächst könnte es so erscheinen, dass das Prinzip der Multiperspektivität mit einer solchen Aneinanderreihung bereits erfüllt sei, ,denn wenn unterschiedliche Standpunkte unter den Tisch fallen, Optionen unterschlagen werden, Alternativen unerörtert bleiben, ist der Weg zur Indoktrination beschritten“ (Wehling 1977, S. 179). Der entscheidende Übergang für politische Bildungsprozesse findet jedoch dann statt, wenn eine individuelle Beurteilung durch Bildungsteilnehmer/-innen möglich wird, beispielweise in einer eigenständigen Beurteilung der Vorannahmen sowie der möglichen Folgen der zur Diskussion stehenden Position. Dann ist der Übergang vom Lernen zur Bildung auch dergestalt möglich, dass die je eigenen Positionierungen aus der Kenntnis anderer, auch entgegenstehender Positionierungen gewonnen werden.

Vor diesem Hintergrund (und im direkten Anschluss an Müller 2016) schlagen wir eine Unterscheidung zwischen additiven und reflexiven Modellen von Multiperspektivität vor. In einer additiven Variante werden zwar unterschiedliche Positionen benannt, aber schlicht nebeneinandergestellt. Im gesellschaftlichen Transfer politikwissenschaftlicher Komplexität können solche Formen den Adressat/-innen auch äußerlich verbleiben: Die eine ist dann dichotom für etwas und der andere dagegen. In einer reflexiven Multiperspektivität wird demgegenüber eine qualitative Befragung der unterschiedlichen Perspektiven angestrebt. Es geht darum, auch entgegenstehende Perspektiven aufzunehmen und einen je eigenständigen, begründeten Umgang damit zu finden. Zum einen können so divergierende Perspektiven mit- und gegeneinander diskutiert werden. Zum anderen wird ein Problem deutlich, das im Anspruch auf Multiperspektivität enthalten sein kann: Das Problem des Relativismus tritt auf, wenn aus Multiperspektivität kurzgeschlossen wird auf eine Beliebigkeit, in der alle Meinungen und Standpunkte unterschiedslos nebeneinander gereiht werden. Ein auf Begründung abzielender Umgang steht einer solchen Beliebigkeit entgegen.

Eng damit verbunden sind normative Vorstellungen, die in allen diskutierten (den eigenen und den anderen) Perspektiven enthalten sind. Nur, wenn auch die eigenen normativen Vorannahmen und Maßstäbe ihrerseits offengelegt werden, können sie der Begründung und der Diskussion zugänglich gemacht werden, um sie zu revidieren, sie zu verbessern oder sie besser zu begründen (Mende 2019, S. 13f.).

Daraus ergeben sich weitere Merkmale für das gesuchte Modell der Übersetzung von Komplexität:

M3) Eine additive Aneinanderreihung unterschiedlicher Positionen verbleibt äuBerlichen Wissensformen stark verhaftet, wie sie im schulischen Kontext etwa in auswendig gelernter, formelhafter Sprache in Klausuren und Prüfungen repräsentiert werden. Reflexive Modelle beziehen neben den divergierenden Positionierungen auch innere Widersprüchlichkeiten und gegenläufige Tendenzen mit ein, die im Rahmen von Bildungsprozessen einer qualitativen Befragung in ihrem Mit-, Gegenund Ineinander ausgesetzt werden.

M4) Zudem zielt ein reflexives Modell auf die Explikation und Offenlegung der eigenen und der anderen normativen Maßstäbe ab, die den jeweiligen Positionierun- 
gen zugrundeliegen. Aus dieser reflexiven Prüfung kann eine erweiterte politische Begründung gewonnen werden, die die jeweils eigene Perspektive revidierbar bzw. begründbar hält, womit auch eine Erweiterung von Denk-, Handlungs- und Urteilsmöglichkeiten einhergeht.

\subsection{Mündigkeit}

Der Anspruch auf Mündigkeit prägt nicht nur fachdidaktische Diskussionen. Spätestens seit den Arbeiten von Theodor W. Adorno, wie sie in der „Erziehung zur Mündigkeit“" vorliegen (Adorno 1971), nehmen die Bezugnahmen auf Mündigkeit in der institutionellen Organisation von Bildungserfahrungen einen zentralen Orientierungspunkt ein.

Die Herausforderung besteht darin, nicht lediglich den bequemen, einfachen (und in der Praxis selteneren) Fall anzunehmen, dass der Wissenstransfer auf umstandslos interessierte und intrinsisch motivierte Bildungsteilnehmer/-innen trifft. Wird der weitaus häufigere und üblichere Weg des Transfers von Wissen an Bildungsteilnehmer/-innen bedacht, dann rückt eine strukturelle Herausforderung genauer in den Blick, nämlich die „Bemühung, Kindern das beizubringen, was sie durchaus nicht lernen wollen“ (Bernfeld 1990, S. 20). An diesem Punkt treten die Stärken der Hinweise Adornos besonders deutlich hervor. Adornos Theorie einer negativen Dialektik ermöglicht es, die Konstellation von Fremd- zur Selbstbestimmung als strukturell grundlegend in institutionell organisierter Bildung anzuerkennen und auszugestalten (Müller 2019). Die Argumentationsfigur der negativen Dialektik erlaubt eine Kritik von autonomieeinschränkenden Voraussetzungen, Annahmen und Folgen der institutionellen Organisation von Bildung und zugleich ein Plädoyer und eine Unterstützung für die autonomieförderlichen Strukturen und Prozesse, die diese für das Subjekt gleichzeitig bereitstellt (Müller 2020). Diese Argumentationsfigur stellt eine Dichotomie von Fremd- oder Selbstbestimmung fundamental in Frage und überschreitet sie, indem die Argumentationsfigur Fremd- zur Selbstbestimmung in Anspruch genommen wird. Momente der Autonomie werden auch in und durch gesellschaftliche Heteronomie ermöglicht, nicht außerhalb oder jenseits davon (Adorno 2001). So argumentiert Adorno nicht per se gegen Fremdbestimmung, sondern er argumentiert aus einer Perspektive der Förderung der Autonomie aller Beteiligten bei gleichzeitiger Kritik all der Momente, die ihr entgegenstehen.

Auch diese Hinweise wollen wir in unser Modell der Übersetzung von Komplexität übernehmen, weil es sich um eine zentrale Annahme im Konzept von Mündigkeit handelt: um die Unterstützung und Förderung des Selber-Denkens anderer. Den fachdidaktischen Diskussionen können so Denkfiguren und Modelle entnommen werden, die an der „Gewinnung eines selbständigen Urteils“ orientiert sind, dieses unterstützen und hervorbringen. Das fünfte Merkmal, das wir den Lösungsmöglichkeiten der Politikdidaktik entnehmen, lautet demnach:

M5) Die Unterstützung und Förderung eines selbständigen Urteils anderer. 


\section{Die Übersetzung von Komplexität in die Gesellschaft}

Die aus der Politischen Bildung gewonnenen Ansprüche korrespondieren damit, dass politikwissenschaftliche Analysen von grundlegenden Kontroversen und Widersprüchen geprägt sind, die sich u. a. aus divergierenden erkenntnis- und wissenschaftstheoretischen Paradigmen sowie normativen Maßstäben speisen. Für unser Modell der Übersetzung von Komplexität ist entscheidend, diese Pluralität und Widersprüchlichkeit in den gesellschaftlichen Transfer einzubeziehen und nicht als problematisch, sondern als produktiv herauszustellen. Es geht uns mithin darum, Komplexität beizubehalten - statt sie abzuschaffen, und sie zu transformieren und $\mathrm{zu}$ übersetzen - statt sie abzubilden.

Nun können die vor bildungstheoretischem Hintergrund und didaktischen Überlegungen entstandenen Lösungsbewegungen nicht unmittelbar in ein Modell der Übersetzung politikwissenschaftlicher Komplexität in die Gesellschaft überführt werden. Die Politikdidaktik unter Bedingungen der Schulpflicht ist an Benotung und Bewertung gebunden und stellt zudem explizit auf die Änderungen von Denk-, Handlungsund Urteilsmöglichkeiten der Bildungsteilnehmer/-innen ab. Die Übersetzung politikwissenschaftlicher Komplexität hingegen stellt Wissen für die Gesellschaft zur Verfügung, das beratend, intervenierend, begleitend oder auch steuernd ausgerichtet sein kann. Sie ist dabei an gänzlich andere Rahmenbedingungen gebunden und bspw. mit den Medien - auch auf wissenschaftsexterne Mechanismen angewiesen. Damit sind grundlegende Differenzen zwischen fachdidaktischen und politikwissenschaftlichen Perspektiven benannt. Vor diesem Hintergrund geht es nun um eine gegenseitige Befragung von Politikwissenschaft und Fachdidaktik, um genauer zu skizzieren, was eine Übersetzung von politikwissenschaftlicher Komplexität in die Gesellschaft (nicht) leisten kann.

\subsection{Bedingungen der Übersetzung von Komplexität}

In inhaltlicher Hinsicht beruht der Anspruch auf Übersetzung politikwissenschaftlicher Komplexität auf den folgenden Merkmalen: M1) das Aushalten politischer Spannungsfelder, M2) die Anerkennung der Eigenständigkeit der Adressat/-innen, M3) eine reflexive, begründende Multiperspektivität, M4) das Offenlegen normativer Maßstäbe und M5) die Organisation eines selbständigen Urteils anderer.

In struktureller Hinsicht zeichnen sich Argumentationsfiguren, die diese inhaltlichen Annahmen auszugestalten erlauben, maßgeblich durch das Aushalten von Widersprüchen aus, die genauer als innere Vermittlungsverhältnisse rekonstruiert werden können. Diese Überlegung ist erläuterungsbedürftig. Argumentiert wurde bislang, dass es bei der Übersetzung politikwissenschaftlicher Komplexität weder um das Vorenthalten noch um eine Verkomplizierung gehen kann, wenn der politikwissenschaftliche und fachdidaktische Anspruch auf Komplexität beibehalten werden soll. Daher ist der Transfer in andere Wissensformen auch maßgeblich von der Eigenlogik der jeweiligen Felder geprägt. Die Fachterminologie der Politikwissenschaft bedient die Politikwissenschaft, die Fachterminologie der Politikdidaktik bedient die Politikdidaktik. Eine zentrale Frage lautet dann, wie die Terminologie, die Mechanismen und Prozesse für den Transfer in die Gesellschaft aussehen 
können. Vor dem Hintergrund der bisherigen Ausführungen wird deutlich, dass es dabei um die Beibehaltung von Komplexität unter den strukturellen Bedingungen der Logik des Feldes geht. Damit rückt eine strukturelle Anforderung deutlicher in den Mittelpunkt: die Aufrechterhaltung und Beibehaltung von Differenzierungen. Diese erschöpfen sich nicht in einem differenzierten Abwägen von pro und contra. Vielmehr bildet sich die Komplexität von Analysen auch darin ab, dass in den einzelnen Momenten auch ihre eventuell unhinterfragten Annahmen, die stillschweigend vorausgesetzten normativen Bezüge und ihre eventuell gegenläufigen Tendenzen beibehalten und beschreibbar gehalten werden. Darauf zielt der Hinweis auf innere Vermittlungsverhältnisse ab. Damit wird über äußerliche Zuordnungen von Annahmen, Analysen, Motiven und Effekten hinausgegangen, indem auch innere Konstitutionsbedingungen einbezogen werden, um eine Differenzierung von (un-)beabsichtigten Annahmen, inneren Widersprüchlichkeiten und Nebenfolgen zu erhalten. Dann werden auch dichotome Modelle überschreitbar, ausdifferenzbar und regen im Transfer in die Gesellschaft eigenständige Denk-, Handlungs- und Urteilsmöglichkeiten an (Müller 2011, 2019).

Dann wird es möglich, politikwissenschaftliche Komplexität weder so zu verkürzen, dass dichotome Lösungsmöglichkeiten als Ausgangsannahme oder als Lösung erscheinen, noch Unterschiede schlicht in ein (relativistisches oder unüberschaubares) Neben- oder Durcheinander aufzulösen. Vielmehr kann von einer Dichotomie auf eine erweiterte Konstellation umgestellt werden, die strukturell als Spannungsfeld konzeptualisiert ist, das äußere und innere Widersprüchlichkeiten und Vermittlungen samt ihrer Begründungen einbezieht.

So zeichnet sich das Modell der Übersetzung von Komplexität dadurch aus, dass über bloße Pro-Contra-Diskussionen hinausgegangen werden kann. Es sammelt nicht lediglich Argumente für den einen oder den anderen Standpunkt, sondern ein differenzierter Erfahrungsraum wird angestrebt, der das In-, Mit- und Gegeneinander der Perspektiven darstellt und aushält.

\subsection{Die Übersetzung unternehmerischer Menschenrechtsverantwortung}

Vor dem Hintergrund unseres Anspruchs auf Offenheit, Kontroversität und Selbständigkeit kann es an dieser Stelle nicht darum gehen, die oben eröffneten Spannungsfelder der unternehmerischen Menschenrechtsverantwortung mit geschlossenen Modellen einzuebnen - nicht zuletzt, weil es in diesem Feld ,nicht die eine richtige Antwort gibt“" (Wetzel 2016, S. 261, Herv. entf.). Stattdessen schlagen wir im Folgenden Perspektiven vor, die ihrerseits geprägt sind von unseren normativen und konzeptuellen Ansätzen und die mit anderen Ansätzen kontrastiert und herausgefordert werden können. Es geht uns an dieser Stelle darum, die Möglichkeiten (und Grenzen) des Modells der Übersetzung von Komplexität zu konturieren.

a) Die Frage einer öffentlichen oder privaten Menschenrechtsverantwortung und ihre Herausforderung durch die Grenzen dieser Dichotomie lässt sich (beispielsweise) in eine Konstellation der gegenseitigen Vermittlung überführen. Privatheit und Öffentlichkeit können als eigenständig und sich gegenseitig konstituierend konzeptualisiert werden: Sie sind ineinander enthalten, weisen aber gleichzeitig eigenständige Logiken auf (ausführlich Mende 2020b; vgl. auch Müller 2011; Ritsert 2017). 
Diese Vermittlungskonstellation kann dahingehend erweitert werden, dass das Modell einer spezifisch unternehmerischen Menschenrechtsverantwortung neue Formen jenseits der Entscheidung zwischen öffentlicher oder privater Verantwortung eröffnet. Solch ein hybrides Modell erlaubt es, das Private und das Öffentliche nicht etwa aufzulösen, sondern beizubehalten, gleichzeitig auszudifferenzieren und durch eine dritte Sphäre zu ergänzen (Mende 2020b, 2020a). Die Herausforderung des öffentlichen Menschenrechtsregimes durch die Macht privater Akteure mündet dann weder in einer dichotomen Entscheidung entweder öffentlicher oder privater Menschenrechtsverantwortung, noch in einer Auflösung der Dichotomie von privat und öffentlich, sondern in einem komplexen, aber nachvollziehbaren Vermittlungsmodell zwischen öffentlichen, privaten und Unternehmens-hybriden Rollen. Auf dieser konzeptuellen Grundlage ist es möglich, neue, hybride Modelle der Unternehmensverantwortung für Menschenrechte zu entwickeln und zu konkretisieren.

Für das Modell der Übersetzung von Komplexität ist an dieser Stelle Folgendes entscheidend: Die auf den ersten Blick (zu) anspruchsvolle Anforderung eines Denkens in Widersprüchen kann in nachvollziehbare Denkfiguren übersetzt werden. Die Anerkennung von (inneren) Widersprüchen führt so weder in Beliebigkeit noch in eine Mystifizierung, sondern sie bietet operationalisierbare Anknüpfungs- und Umgangsmöglichkeiten auf der Grundlage von Komplexität.

b) Die Frage für oder gegen einen neuen völkerrechtlichen Vertrag lässt sich überführen in eine Perspektive, die die Komplexität der Debatte anerkennt (die auch von einer Vielzahl der Debattenteilnehmer/-innen betont wird) -, ohne dabei die Notwendigkeit politischen Handelns aus dem Blick zu verlieren. Ein Weg besteht darin, die jeweiligen Gründe und Begründungen der Pro-, Contra- und gemischten Positionen auf- und ernstzunehmen, um auf dieser Basis nach Lösungswegen zu suchen. Dieser Weg erlaubt eine Erweiterung der Perspektive jenseits von dichotomen Bekenntnissen für oder gegen eine bestimmte Form der Unternehmensverantwortung. Er ermöglicht die Offenlegung von Gründen, Hintergründen und Begründungen statt einer Proklamation von Standpunkten. Gleichzeitig erzwingt er diese Begründungen auch. Rein strategische Manöver (der Vereinfachung oder der Abbildung von Komplexität) werden zugunsten der inhaltlichen und deliberativen Auseinandersetzung problematisiert. Daraus ergibt sich eine Perspektivenvielfalt, die eine Auseinandersetzung mit pluralen und auch mit entgegenstehenden Argumenten nahezu erfordert - selbst wenn es ,lediglich“ darum geht, die jeweils eigene Begründung in der Auseinandersetzung mit anderen Begründungen zu schärfen. Das ermöglicht eine Beschäftigung mit den Inhalten und den Konsequenzen eines völkerrechtlichen Vertrags, die sowohl dessen menschenrechtsstärkende als auch dessen problematische Aspekte in ihrer Gleichzeitigkeit in den Blick nehmen kann. Jenseits des Bekenntniszwangs sind dann auch Fragen danach möglich, inwiefern bspw. der UN-Vertragsprozess mit dem existierenden Konsens der UN-Leitprinzipien kompatibel ist, inwiefern er den Blick auf weiterhin bestehende Lücken im Menschenrechtsregime lenkt und inwiefern er in der Lage ist, diese Lücken tatsächlich zu schließen - sowohl in völkerrechtlicher als auch in politischer Hinsicht. Denn selbst ein deliberativ vorbildlich geführter Prozess ist letztlich auf die Zustimmung und Unterstützung von Staaten angewiesen, die den Vertrag befürworten, ratifizieren und implementieren. 
Zentral für die Übersetzung von Komplexität ist hier zweierlei: Erstens gehen Begründungen über Setzungen hinaus. Statt eine Antwort (oder in einer additiven Perspektive zwei oder drei Antworten) lediglich vorzugeben, öffnen Begründungen den Blick auf Implikationen, die Gebundenheit an strukturelle Bedingungen, (un)beabsichtigte Effekte und die (stillschweigend) in Anspruch genommenen normativen Maßstäbe. Das ermöglicht zweitens eine Positionierung aufseiten der Adressat/ -innen, die ihrerseits begründet statt gesetzt werden kann. Dadurch zeichnet sich eine Umstellung von einer Meinung zu einer Begründung ab, ohne die Perspektivenvielfalt einzuschränken. In der gelungenen Variante wird diese Perspektivenvielfalt integriert in ein produktives und umsetzbares Ergebnis. Diese Variante ist jedoch keineswegs garantiert: nicht zuletzt, weil neben Begründungslogiken und deren Widersprüchen auch Logiken der Macht und des Eigeninteresses eine Rolle spielen.

c) Wenn dichotome und personalisierende Zuschreibungen von guten oder schlechten Unternehmen aufgegeben werden, öffnet sich die Perspektive für das Denken jenseits von Dichotomien. Damit geht eine Perspektive einher, die Unternehmen als handlungsfähige Akteure in der Global Governance weder dämonisiert noch glorifiziert, sondern zunächst als empirische Gegebenheit anerkennt, mit der das Menschenrechtsregime einen Umgang finden muss. Damit ist bereits wieder ein normativer Maßstab eingeführt, nämlich das Bestreben, Menschenrechte zu stärken. Vor diesem normativen Hintergrund lassen sich konkrete Unternehmenshandlungen, in gewissen Fällen auch ganze Unternehmen, normativ diskutieren. Deutlich wird dann zum einen, wie nah beieinander menschenrechtsverletzende und -bewahrende Effekte mitunter liegen können. Zum anderen zeigt sich dann auch die hohe Pluralität und Heterogenität der Unternehmen, ihrer Handlungsfähigkeit und ihrer Effekte. Etwaige Regulierungsmaßnahmen und Instrumente lassen sich vor diesem Hintergrund nicht nur normativ diskutieren, sondern auch entlang anderer Differenzlinien zwischen Unternehmen ausdifferenzieren. Die oben genannten Konzepte der Komplizenschaft, der Rechenschaftspflicht oder der unternehmerischen Einflussmöglichkeiten (leverage) bieten zentrale Dreh- und Angelpunkte, um Dichotomien umzustellen auf ein Denken in Konstellationen, Widersprüchen und inneren Vermittlungsverhältnissen. Im Feld der unternehmerischen Menschenrechtsverantwortung werden sie bereits diskutiert. Die Umstellung der Dichotomie zwischen „guten“ und ,schlechten“ Unternehmen auf die Öffnung des Blicks für das In-, Mit- und Gegeneinander, für Widersprüche und Differenzierungen bietet eine Grundlage dafür, komplexe politikwissenschaftliche Analysen in die Gesellschaft $\mathrm{zu}$ übersetzen.

Für unser Modell der Übersetzung von Komplexität sind bereits weit unterhalb dieser Figur, nämlich mit einer klein- und feinteiligeren Sortierungsarbeit in „gute" und „schlechte“ Dimensionen von Praktiken, Mechanismen und Prozesse drei konkrete Effekte verbunden: Erstens gibt sie das Modell einer personalisierenden Zuschreibung und Kritik zugunsten einer differenzierteren Perspektive auf und kann so die Gleichzeitigkeit der Dimensionen im Sinne einer reflexiven Multiperspektivität anerkennen. Zweitens eröffnet sie den Blick auf Implikationen, unterschiedliche Effekte derselben Handlung oder etwa Unterschiede zwischen Intentionen und Auswirkungen. Drittens wird auch die Perspektive auf unterschiedliche normative Maß- 
stäbe geöffnet, die wiederum eine Begründungspflicht für jeweilige Bewertungen verdeutlicht. Aufgelöst werden nicht normative Bewertungen, sondern ihre Setzung. An ihre Stelle treten Begründungen.

\section{Drei Modelle der Komplexität: Möglichkeiten und Grenzen}

Die drei Modelle der Vereinfachung, der Abbildung und der Übersetzung von Komplexität lassen sich nun bezüglich ihrer Möglichkeiten und Grenzen idealtypisch folgendermaßen skizzieren (vgl. Tab. 1).

Die Vereinfachung von Komplexität hat bessere Möglichkeiten, gesellschaftlich und politisch Gehör zu finden. Sie entspricht den Bedingungen der Markt- und Verkaufsförmigkeit von Wissen am besten. Ihre Grenzen bestehen darin, Sachverhalte $\mathrm{zu}$ verkürzen und Wissen der Gesellschaft vorzuenthalten.

Die Abbildung von Komplexität erkennt die Relevanz von Komplexität für Wissen und Wissenstransfer an, kann aber in einem additiven oder relativistischen Nebeneinanderstellen pluraler Perspektiven verharren. Zudem vermischt sie unterschiedliche Logiken von Politikwissenschaft und Gesellschaft. Wissen kann hier verkompliziert werden und damit auch einen Grad an Unzugänglichkeit generieren.

Das Modell der Übersetzung von Komplexität strebt an, Komplexität für die Beteiligten nachvollziehbar aufzubereiten und darzustellen, um politikwissenschaftliches Wissen ohne Reduktion gesellschaftlich zugänglich zu machen. Seine Herausforderung besteht in der Eigenständigkeit der Übersetzungsarbeit, für die Prozesse, Mechanismen und ggf. auch Terminologien gefunden werden müssen. Nicht zuletzt aufgrund der Eigenlogiken in Politik und Gesellschaft sowie aufgrund der Autonomie der Adressat/-innen steht das Modell auch vor der Herausforderung, dass es keine gesicherten Garantien für den gelungenen Transfer politikwissenschaftlicher Komplexität in die Gesellschaft gibt. Ein Grund dafür besteht auch darin, dass dem Verständnis von ,gelingender“ Übersetzung unterschiedliche Ansprüche und norma-

Tab. 1 Drei Modelle des gesellschaftlichen Transfers politikwissenschaftlicher Komplexität

\begin{tabular}{|c|c|c|c|}
\hline $\begin{array}{l}\text { Transfer } \\
\text { von Kom- } \\
\text { plexität }\end{array}$ & Möglichkeiten & Problematiken & Folgen \\
\hline Vereinfachung & $\begin{array}{l}\text { Gehör in der Gesell- } \\
\text { schaft; } \\
\text { Präsentation ,einfacher“ } \\
\text { Lösungen }\end{array}$ & $\begin{array}{l}\text { Verkürzung von Inhalten und Lösun- } \\
\text { gen }\end{array}$ & $\begin{array}{l}\text { Wissen und } \\
\text { Analyse werden } \\
\text { vorenthalten }\end{array}$ \\
\hline Abbildung & $\begin{array}{l}\text { Komplexität wird beibe- } \\
\text { halten }\end{array}$ & $\begin{array}{l}\text { Relativismus; } \\
\text { additiver Pluralismus; } \\
\text { Gleichsetzung unterschiedlicher } \\
\text { Logiken (Kategorienfehler) }\end{array}$ & $\begin{array}{l}\text { Wissen und } \\
\text { Analyse werden } \\
\text { verkompliziert }\end{array}$ \\
\hline Übersetzung & $\begin{array}{l}\text { Nachvollziehbarkeit von } \\
\text { Komplexität; } \\
\text { Komplexität als Start- } \\
\text { punkt }\end{array}$ & $\begin{array}{l}\text { Übersetzungsarbeit; } \\
\text { keine Garantien }\end{array}$ & $\begin{array}{l}\text { Wissen und } \\
\text { Analyse werden } \\
\text { aufbereitet }\end{array}$ \\
\hline
\end{tabular}

Bedingungen der Verwertbarkeit von Wissen 
tive Vorstellungen zugrundeliegen können, die sich ihrerseits nicht einfach setzen lassen.

Insgesamt sind alle drei Modelle sowohl mit Möglichkeiten als auch mit Problematiken verbunden. Hinzu kommt eine weitere Ebene, die die Modelle prägt. Sie alle stehen vor der Herausforderung, politikwissenschaftliche Komplexität in das Feld der Gesellschaft zu transferieren, das von anderen Logiken gekennzeichnet ist: Wissen und Komplexität dienen hier nicht vorrangig dem Austausch und Gewinn von Erkenntnissen und Forschungsergebnissen, sondern der politischen, der medialen, der machterhaltenden und/oder der marktförmigen Verwertbarkeit. ${ }^{8}$

An diesem Punkt sehen wir eine entscheidende Stärke des Modells der Übersetzung von Komplexität, die einen Startpunkt für gesellschaftliches Denken und Handeln schafft. Dieser Aspekt kann auch der Diskussion um die Unternehmensverantwortung für Menschenrechte entnommen werden. Die Befürworter/-innen der Weiterentwicklung von Unternehmensverantwortung im UN-Vertragsprozess benennen Komplexität als Herausforderung, für die Lösungen gefunden werden müssen, während die ablehnenden Positionen Komplexität von vornherein als Grund für das Scheitern jeglicher Vertragsbemühungen setzen. Beide Perspektiven erkennen Komplexität an: die einen als Endpunkt, die anderen als Startpunkt.

Der Anspruch auf Komplexität als Startpunkt wird mit der Vorwegnahme einfacher, vorgegebener oder additiver Lösungen unterlaufen. Diese stehen der Förderung und Unterstützung des eigenständigen Denkens anderer entgegen. Dagegen können Offenheit, Kontroversität und Multiperspektivität Neugier produzieren und das Suchen und Debattieren von Umgangsmöglichkeiten motivieren. Das ist wiederum eine gelungene Variante der Übersetzung politikwissenschaftlicher Komplexität in die Gesellschaft, die (so wie alle anderen Modelle) auch andere Verläufe nehmen kann. Kausalaussagen sind im Transfer politikwissenschaftlicher Komplexität in die Gesellschaft gerade nicht möglich - und müssen dies im dritten Modell auch gar nicht sein. Denn das Modell der Übersetzung von Komplexität konzeptualisiert alle Beteiligten so, dass sie in ihrer Eigenständigkeit anerkannt werden, auch wenn gegensätzliche Positionierungen vorliegen. Letztlich kann somit dasjenige Modell, das weniger Sicherheit garantiert, mit mehr Sicherheit politikwissenschaftliches Wissen in der Gesellschaft und damit auch gesellschaftliches Wissen, Denken und Handeln ausbauen und vertiefen.

Funding Open Access funding provided by Projekt DEAL.

Open Access Dieser Artikel wird unter der Creative Commons Namensnennung 4.0 International Lizenz veröffentlicht, welche die Nutzung, Vervielfältigung, Bearbeitung, Verbreitung und Wiedergabe in jeglichem Medium und Format erlaubt, sofern Sie den/die ursprünglichen Autor(en) und die Quelle ordnungsgemäß nennen, einen Link zur Creative Commons Lizenz beifügen und angeben, ob Änderungen vorgenommen wurden.

Die in diesem Artikel enthaltenen Bilder und sonstiges Drittmaterial unterliegen ebenfalls der genannten Creative Commons Lizenz, sofern sich aus der Abbildungslegende nichts anderes ergibt. Sofern das betref-

\footnotetext{
8 Auch wenn die Politikwissenschaft mit Drittmittelerfolgen oder fachwissenschaftlicher Reputation ebenfalls Kriterien der Verwertbarkeit kennt und es trennscharf „die“ politikwissenschaftliche Logik ebenso wenig gibt wie „die“ gesellschaftliche, geht es uns hier darum, dass sich die Felder und ihre jeweiligen Logiken und Mechanismen voneinander unterscheiden.
} 
fende Material nicht unter der genannten Creative Commons Lizenz steht und die betreffende Handlung nicht nach gesetzlichen Vorschriften erlaubt ist, ist für die oben aufgeführten Weiterverwendungen des Materials die Einwilligung des jeweiligen Rechteinhabers einzuholen.

Weitere Details zur Lizenz entnehmen Sie bitte der Lizenzinformation auf http://creativecommons.org/ licenses/by/4.0/deed.de.

\section{Literatur}

Addo, Michael K. 2014. The reality of the United Nations guiding principles on business and human rights. Human Rights Law Review 14(1):133-147.

Adorno, Theodor W. 1971. Erziehung zur Mündigkeit: Vorträge und Gespräche mit Hellmut Becker 1959-1969. Frankfurt am Main: Suhrkamp.

Adorno, Theodor W. 2001. Zur Lehre von der Geschichte und von der Freiheit. Frankfurt am Main: Suhrkamp.

Atal, Maha Rafi. 2017. White capital: corporate social responsibility and the limits of transformation in South Africa. The Extractive Industries and Society 4(4):735-743.

Behrmann, Günter, Tilman Grammes, und Sibylle Reinhardt. 2004. Politik. Kerncurriculum Sozialwissenschaften in der gymnasialen Oberstufe. In Kerncurriculum für die Oberstufe II - Biologie, Chemie, Physik, Geschichte, Politik, Hrsg. Heinz-Elmar Tenorth, 322-406. Weinheim u. a.: Beltz.

Bernfeld, Siegfried. 1990. Sisyphos oder die Grenzen der Erziehung. Frankfurt am Main: Suhrkamp.

von Bernstorff, Jochen. 2011. Extraterritoriale menschenrechtliche Staatenpflichten und Corporate Social Responsibility: Wie weit geht die menschenrechtliche Verantwortung des Staates für das Verhalten eigener Unternehmen im Ausland? Archiv des Völkerrechts 49(1):34-63.

Bonfadelli, Heinz, Birte Fähnrich, Corinna Lüthje, Jutta Milde, Markus Rhomberg, und Mike S. Schäfer (Hrsg.). 2017. Forschungsfeld Wissenschaftskommunikation. Wiesbaden: Springer VS.

Clapham, Andrew. 2006. Human rights obligations of non-state actors. Oxford, New York: Oxford University Press.

Dernbach, Beatrice, Christian Kleinert, und Herbert Münder (Hrsg.). 2013. Handbuch Wissenschaftskommunikation. Wiesbaden: VS.

Deva, Surya, und David Bilchitz (Hrsg.). 2013. Human rights obligations of business: beyond the corporate responsibility to respect? Cambridge: Cambridge University Press.

Fitzgerald, Oonagh. 2019. Addressing the human rights conduct of transnational corporations through international institutional bypasses. Transnational Legal Theory https://doi.org/10.1080/20414005. 2019.1651123.

Hsieh, Nien. 2015. Should business have human rights obligations? Journal of Human Rights 14(2):218236.

Mende, Janne. 2015. Kultur als Menschenrecht?: Ambivalenzen kollektiver Rechtsforderungen. Frankfurt am Main, New York: Campus.

Mende, Janne. 2017. Privatisierung oder Diffusion von Verantwortung?: Die Entwicklung wirtschaftlicher Verantwortung für Menschenrechte. In Politik und Verantwortung Politische Vierteljahresschrift, Bd. Sonderheft 52, Hrsg. Christopher Daase, Julian Junk, Stefan Kroll, und Valentin Rauer, 409-435.

Mende, Janne. 2019. Are human rights western-And why does it matter? A perspective from international political theory. Journal of International Political Theory https://doi.org/10.1177/ 1755088219832992.

Mende, Janne. 2020a. Business authority in global governance: beyond public and private. WZB Berlin social science center discussion paper, Bd. SP IV 2020-103 https://bibliothek.wzb.eu/pdf/2020/iv20103.pdf.

Mende, Janne. 2020b. The public, the private, and the business-societal: a threefold approach to business responsibility for human rights. In Research agendas for human rights, Hrsg. Alison Brysk, Michael Stohl. Cheltenham: Edward Elgar. im Erscheinen.

Miretski, Pini Pavel, und Sascha-Dominik Bachmann. 2014. The "UN Norms on the Responsibility of Transnational Corporations and Other Business Enterprises with Regard to Human Rights": a requiem. Deakin Law Review 17(1):5-41.

Muchlinski, Peter. 2012. Implementing the new UN corporate human rights framework. Business Ethics Quarterly 22(1):145-177.

Müller, Stefan. 2011. Logik, Widerspruch und Vermittlung: Aspekte der Dialektik in den Sozialwissenschaften. Wiesbaden: VS. 
Müller, Stefan. 2016. Multiperspektivität und Reflexivität als Bezugspunkte politischer Bildung. Zeitschrift für Didaktik der Gesellschaftswissenschaften 7(2):108-118.

Müller, Stefan. 2018. Rechthaberei und Reflexion. Sozialwissenschaftliche Modelle und Möglichkeiten von Kritik. Zeitschrift für Didaktik der Gesellschaftswissenschaften 9(1):113-131.

Müller, Stefan. 2019. Mündigkeit. Zwei Argumente für eine reflexive, nicht-dichotome Perspektive. In Gesellschaft im Wandel - Neue Aufgaben für die politische Bildung und ihre Didaktik!?, Hrsg. Kerstin Pohl, Matthias Lotz, 86-93. Frankfurt am Main: Wochenschau-Verlag.

Müller, Stefan. 2020. Das Versprechen vom Bessermachen. Reflexion und Kritik im Kontext institutioneller Bildung. Inter- und transdisziplinäre Bildung 2(2):6-15. https://www.itdb.ch/index.php/itdb/ article/view/24.

Noortmann, Math, August Reinisch, und Cedric Ryngaert (Hrsg.). 2015. Non-state actors in international law. Oxford: Hart.

Ramasastry, Anita. 2015. Corporate social responsibility versus business and human rights: bridging the gap between responsibility and accountability. Journal of Human Rights 14(2):237-259.

Reinhardt, Sibylle. 2018. Politik-Didaktik: Praxishandbuch für die Sekundarstufe I und II. Berlin: Cornelsen.

Ritsert, Jürgen. 2017. Summa Dialectica: Ein Lehrbuch zur Dialektik. Weinheim/Basel: Beltz Juventa.

Ruggie, John G. 2018. Die soziale Konstruktion der Leitlinien für Wirtschaft und Menschenrechte der Vereinten Nationen. Leviathan 46(1):6-36.

Ruggie, John Gerard, und John F. Sherman. 2017. The concept of "due diligence" in the UN Guiding Principles on Business and Human Rights: a reply to Jonathan Bonnitcha and Robert McCorquodale. European Journal of International Law 28(3):921-928.

Sander, Wolfgang. 2017. Der Beutelsbacher Konsens zwischen Theorie und Praxis der politischen Bildung. In Der Beutelsbacher Konsens. Bedeutung, Wirkung, Kontroversen, Hrsg. Siegfried Frech, Dagmar Richter, 57-68. Schwalbach/Ts.: Wochenschau-Verlag.

Scherer, Andreas Georg, und Guido Palazzo. 2011. The new political role of business in a globalized world: a review of a new perspective on CSR and its implications for the firm, governance, and democracy. Journal of Management Studies 48(4):899-931.

Tuttle, Nicole R. 2015. Human Rights Council resolutions 26/9 and 26/22: Towards corporate accountability? Insights 19 (20). https://www.asil.org/insights/volume/19/issue/20/human-rights-councilresolutions-269-and-2622-towards-corporate\#_ednref5. Zugegriffen: 31. Dez. 2015.

UN. 2011. Guiding principles on business and human rights: implementing the United Nations ,protect, respect and remedy " framework. New York, Geneva: United Nations.

Wehling, Hans-Georg. 1977. Konsens à la Beutelsbach? Nachlese zu einem Expertengespräch. In Das Konsensproblem in der politischen Bildung, Hrsg. Siegfried Schiele, Herbert Schneider, 173-184. Stuttgart: Klett.

Wettstein, Florian. 2012. Silence as complicity: elements of a corporate duty to speak out against the violation of human rights. Business Ethics Quarterly 22(1):37-61.

Wetzel, Julia Ruth-Maria. 2016. Human rights in transnational business: translating human rights obligations into compliance procedures. Cham: Springer.

Wood, Stepan. 2012. The case for leverage-based corporate human rights responsibility. In Business and human rights, Hrsg. Wesley Cragg, 135-177. Cheltenham: Edward Elgar.

Černič, Jernej Letnar, und Nicolás Carrillo Santarelli (Hrsg.). 2018. The future of business and human rights: theoretical and practical considerations for a UN treaty. Cambridge: Intersentia. 